\title{
On some properties of the space of upper semicontinuous functions
}

\author{
Alexander V. Osipov ${ }^{\mathrm{a}}$, Evgenii G. Pytkeev ${ }^{\mathrm{b}}$ \\ ${ }^{a}$ Krasovskii Institute of Mathematics and Mechanics, Ural Federal University, Ural State \\ University of Economics, 620219, Ekaterinburg, Russia \\ ${ }^{b}$ Krasovskii Institute of Mathematics and Mechanics, Ural Federal University, 620219, \\ Ekaterinburg, Russia
}

\begin{abstract}
For a Tychonoff space $X$, we will denote by $U S C_{p}(X)\left(B_{1}(X)\right)$ the set of all real-valued upper semicontinuous functions (the set of all Baire functions of class 1 ) defined on $X$ endowed with the pointwise convergence topology.

In this paper we describe a class of Tychonoff spaces $X$ for which the space $U S C_{p}(X)$ is sequentially separable. Unexpectedly, it turns out that this class coincides with the class of spaces for which a stronger form of the sequential separability for the space $B_{1}(X)$ holds.
\end{abstract}

Keywords: sequentially separable, function space, continuous function, upper semicontinuous function, Baire function class 1

2010 MSC: 54C35, 54C30, 54A20, 54H05

\section{Introduction}

If $X$ is a topological space and $A \subseteq X$, then the sequential closure of $A$, denoted by $[A]_{\text {seq }}$, is the set of all limits of sequences from $A$. A set $D \subseteq X$ is said to be sequentially dense if $X=[D]_{\text {seq }}$. If $D$ is a countable sequentially dense subset of $X$ then $X$ is called sequentially separable space [7, 9].

Let $X$ be a Tychonoff space. We consider the following function spaces.

- $C_{p}(X)$ is the set of all real-valued continuous functions defined on $X$ endowed with the topology of pointwise convergence.

URL: OAB@list.ru (Alexander V. Osipov), pyt@imm.uran.ru (Evgenii G. Pytkeev) 
- $B_{1}(X)$ is the set of all Baire functions class 1 (i.e., pointwise limits of continuous functions) defined on $X$ endowed with the pointwise convergence topology.

- $U S C_{p}(X)$ is the set $U S C(X)=\left\{f \in \mathbb{R}^{X}: f^{-1}((-\infty, r))\right.$ is an open set of $X$ for any $r \in \mathbb{R}\}$ (i.e. the set all upper semicontinuous functions defined on $X$ ) endowed with the topology of pointwise convergence.

Note that $C_{p}(X) \subseteq U S C_{p}(X) \subseteq B_{1}(X)$ for a separable metrizable space $X$. It follows that $U S C_{p}(X)$ is sequentially separable for a separable metrizable space $X$ (Theorem 3.2).

It is well known that $f: X \rightarrow \mathbb{R}$ is a Baire function if and only if there exists a continuous mapping $\varphi: X \rightarrow M$ from $X$ onto a separable metrizable space $M$ and a Borel function $g: M \rightarrow \mathbb{R}$ such that $f=g \circ \varphi$. If we replace the Borel function by upper semicontinuous function in this characterization, we obtain the function $f: X \rightarrow \mathbb{R}$ such that $f^{-1}((-\infty, r))=\varphi^{-1}\left(g^{-1}((-\infty, r))\right)$ is a cozero-set of $X$ for any $r \in \mathbb{R}$.

Define the function space $U S C^{f}(X):=\left\{f \in \mathbb{R}^{X}: f^{-1}((-\infty, r))\right.$ is a cozero set of $X$ for any $r \in \mathbb{R}\}$. Clearly, that if $X$ is a perfectly normal space then $U S C^{f}(X)=U S C(X)$. We denote by $U S C_{p}^{f}(X)$ the set $U S C^{f}(X)$ endowed with the topology of pointwise convergence.

We claim that $U S C_{p}^{f}(X)$ is sequentially separable if and only if there exists a countable subset $S$ of $C(X)$ such that $[S]_{\text {seq }}=B_{1}(X)$ (i.e., when a stronger form of the sequential separability for the space $B_{1}(X)$ holds).

\section{Main definitions and notation}

We recall that a subset of $X$ that is the complete preimage of zero for a certain function from $C(X)$ is called a zero-set. A subset $O \subseteq X$ is called a cozero-set of $X$ if $X \backslash O$ is a zero-set. If a set $Z=\bigcup_{i \in \mathbb{N}} Z_{i}$ where $Z_{i}$ is a zero-set of $X$ for any $i \in \mathbb{N}$, then $Z$ is called $Z_{\sigma}$-set of $X$. Note that if a space $X$ is a perfectly normal space, then class of $Z_{\sigma^{-}}$sets of $X$ coincides with class of $F_{\sigma}$-sets of $X$. It is well known [6], that $f \in B_{1}(X)$ if and only if $f^{-1}(G)$ $Z_{\sigma}$-set for any open set $G$ of real line $\mathbb{R}$.

Recall that the $i$-weight $i w(X)$ of a space $X$ is the smallest infinite cardinal number $\tau$ such that $X$ can be mapped by a one-to-one continuous mapping onto a Tychonoff space of the weight not greater than $\tau$.

Theorem 2.1. (Noble's Theorem in [0]) Let $X$ be a Tychonoff space. A space $C_{p}(X)$ is separable if and only if iw $(X)=\aleph_{0}$. 
Theorem 2.2. (Pestriakov's Theorem in [5]). Let $X$ be a Tychonoff space. A space $B_{1}(X)$ is separable if and only if iw $(X)=\aleph_{0}$.

Definition 2.3. A Tychonoff space $X$ has the Velichko property $(X \models V)$, if there exists a condensation (one-to-one continuous mapping) $f: X \mapsto Y$ from the space $X$ on a separable metric space $Y$, such that $f(U)$ is an $F_{\sigma}$-set of $Y$ for any cozero-set $U$ of $X$.

Theorem 2.4. (Velichko [8] ). Let $X$ be a Tychonoff space. A space $C_{p}(X)$ is sequentially separable if and only if $X \models V$.

Theorem 2.5. ([8]) A space $B_{1}(X)$ is sequentially separable for any separable metric space $X$.

Note that proof of this theorem gives more, namely that there exists a countable subset $S \subset C_{p}(X)$, such that $[S]_{s e q}=B_{1}(X)$.

Hence, a space $U S C_{p}(X)$ is sequentially separable for any separable metric space $X$.

In [4], Osipov and Pytkeev have established criterion for $B_{1}(X)$ to be sequentially separable.

Definition 2.6. A space $X$ has $O P$-property $(X \models O P)$, if there exists a bijection $\varphi: X \mapsto Y$ from a space $X$ onto a separable metrizable space $Y$, such that

1. $\varphi^{-1}(U)$ is a $Z_{\sigma^{-}}$-set of $X$ for any open set $U$ of $Y$;

2. $\varphi(T)$ is an $F_{\sigma}$-set of $Y$ for any zero-set $T$ of $X$.

Theorem 2.7. (Theorem 3.1 in [4]) A function space $B_{1}(X)$ is sequentially separable if and only if $X \models O P$.

Theorem 2.8. (Example 3.3 in [4]) There is a Tychonoff space $X$ such that $C_{p}(X)$ is sequentially separable, but $B_{1}(X)$ is not.

In the above theorem, the promised space $X$ could be, for example, if $X$ is the Sorgenfrey line (or the Niemytzki plane) [4]. 


\section{Main results}

Definition 3.1. A space $X$ has $U$-property $(X \models U)$, if there exists a condensation $f: X \mapsto Y$ from the space $X$ onto a separable metric space $Y$, such that $f(D)$ is a $Z_{\sigma}$-set of $Y$ for any zero-set $D$ of $X$.

Theorem 3.2. Let $X$ be a Tychonoff space. Then the following statements are equivalent:

1. $U S C_{p}^{f}(X)$ is sequentially separable;

2. $X \models U$;

3. there exists a countable subset $S$ of $C(X)$ such that $[S]_{\text {seq }}=B_{1}(X)$.

Proof. (1) $\Rightarrow(2)$. Assume that $U S C_{p}^{f}(X)$ is sequentially separable. Let $A=\left\{f_{i}: i \in \mathbb{N}\right\}$ be a sequentially dense subset of $U S C_{p}^{f}(X)$. Note that $f^{-1}(W)$ is a $Z_{\sigma}$-set of $X$ for an open set $W$ of $\mathbb{R}$ and $f \in U S C_{p}^{f}(X)$. It follows that $U S C_{p}^{f}(X)$ is a dense subset of $B_{1}(X)$ and hence $B_{1}(X)$ is separable. By Theorem $\left[2.2\right.$, $i w(X)=\aleph_{0}$. Hence there exists a condensation from the space $X$ on a separable metric space $M$. Let $\beta$ be a countable base of the space $M$. Let $\alpha=\left\{f_{i}^{-1}(-\infty, r): r \in \mathbb{Q}\right.$ and $\left.i \in \mathbb{N}\right\} \bigcup \beta$ and let $\tau$ be a topology on $X$ generating $\alpha$. Denote $Y=(X, \tau)$. Note that there exists a condensation $f: X \mapsto Y$ from the space $X$ onto a separable metric space $Y$. By definition of $\alpha, f_{i} \in U S C(Y)$ for each $i \in \mathbb{N}$.

We will prove that $f(D)$ is an $F_{\sigma}$-set of $Y$ for any $Z_{\sigma}$-set $D$ of $X$. Fix a $Z_{\sigma}$-set $D=\bigcup_{i \in \mathbb{N}} D_{i}$ of $X$ where $D_{i}$ is a zero-set of $X$ and $D_{i} \subset D_{i+1}$ for each $i \in \mathbb{N}$. Define the function $h: h\left(D_{1}\right)=1, h\left(D_{i+1} \backslash D_{i}\right)=\frac{1}{i+1}$ for each $i \in \mathbb{N}$ and $h(X \backslash D)=0$. By construction of $h, D=h^{-1}((0,+\infty))$.

Note that $h \in U S C^{f}(X)$ and hence there are $\left\{f_{i_{k}}: k \in \mathbb{N}\right\} \subset A$ such that $f_{i_{k}} \rightarrow h(k \rightarrow \infty)$. It follows that $D=h^{-1}((0,+\infty))=\bigcup_{j \in \mathbb{N}} \bigcap_{i_{k}>j} f_{i_{k}}^{-1}\left(\left[\frac{1}{j},+\infty\right)\right)$ and hence $f(D)$ is an $F_{\sigma}$-set of $Y$.

$(2) \Rightarrow(1)$. Assume that $X \models U$, i.e. there is a condensation (oneto-one continuous mapping) $f: X \mapsto Y$ from the space $X$ on a separable metric space $Y$, such that $f(D)$ is an $F_{\sigma}$-set of $Y$ for any $Z_{\sigma}$-set $D$ of $X$. Then $U S C_{p}^{f}(X) \subset U S C_{p}(Y) \subset B_{1}(Y)$. By Velichko's Theorem 2.5, there is $A=\left\{f_{i}: i \in \mathbb{N}\right\} \subset C_{p}(Y)$ such that $[A]_{\text {seq }}=B_{1}(Y)$. Note that $C_{p}(Y) \subset$ $C_{p}(X) \subset U S C_{p}^{f}(X) \subset B_{1}(Y)$. It follows that $A$ is a countable sequentially dense subset of $U S C_{p}^{f}(X)$. 
$(3) \Rightarrow(2)$. Suppose that exists a countable subset $S$ of $C(X)$ such that $[S]_{\text {seq }}=B_{1}(X)$. Consider a topology $\tau$ generated by the family $\alpha=$ $\left\{f^{-1}(G): G\right.$ is an open subset of $\mathbb{R}$ and $\left.f \in S\right\}$. Denote $Y=(X, \tau)$. Let $\varphi$ be a identity map from $X$ onto $Y$. By Theorem 3.1 in [4], $\varphi$ is a bijection such that $\varphi(D)$ is a $Z_{\sigma}$-set of $Y$ for any zero-set $D$ of $X$. Since $S \subset C(X)$, $\varphi$ is a condensation.

$(2) \Rightarrow(3)$. Let $X \models U$. By Theorem 2.5, there exists a countable dense subset $L$ of $C_{p}(Y)$ such that $[L]_{s e q}=B_{1}(Y)$. Then $S=\{f \circ \varphi: f \in L\}$ is a countable subset of $C(X)$. Let $\varphi^{*}(h):=h \circ \varphi$ for $h \in B_{1}(Y)$. Then $\varphi^{*}: B_{1}(Y) \mapsto B_{1}(X)$ is a first-level Baire isomorphism. It follows that $[S]_{s e q}=B_{1}(X)$.

Corollary 3.3. Let $X$ be a Tychonoff space and let $U S C_{p}^{f}(X)$ be sequentially separable. Then $C_{p}(X)$ and $B_{1}(X)$ are sequentially separable.

Proof. By Theorem $2.4, C_{p}(X)$ is sequentially separable. By Theorem 2.7. $B_{1}(X)$ is sequentially separable.

Corollary 3.4. Let $X$ be a perfectly normal space. Then the following statements are equivalent:

1. $U S C_{p}(X)$ is sequentially separable;

2. $X \models U$;

3. there exists a countable subset $S$ of $C(X)$ such that $[S]_{\text {seq }}=B_{1}(X)$.

A continuous image of sequentially separable space is sequentially separable. Hence cosmic spaces - the continuous images of separable metric spaces (space with a countable network) - are sequentially separable. So, for any separable metric space $X$ (or more generally, cosmic $X), C_{p}(X)$ is cosmic, and hence sequentially separable.

Corollary 3.5. Let $X$ be a separable metrizable space. Then:

1. $U S C_{p}(X)$ is sequentially separable;

2. $B_{1}(X)$ is sequentially separable;

3. there exists a countable subset $S$ of $C(X)$ such that $[S]_{\text {seq }}=B_{1}(X)$. 
Recall that an analytic space is a metrizable space that is a continuous image of a Polish space.

A map $f: X \rightarrow Y$ be called $Z_{\sigma}$-map, if $f^{-1}(Z)$ is a $Z_{\sigma^{-}}$set of $X$ for any zero-set $Z$ of $Y$.

We need the following theorem as a special case of the Theorem 1 in [1].

Theorem 3.6. ([1] $]$ Suppose that $\varphi: L \mapsto S$ be a $Z_{\sigma}$-mapping from an analytic space $L$ onto a cosmic space $S$. Then $\varphi$ is piecewise continuous.

Theorem 3.7. There exists a Tychonoff space $X$ such that $C_{p}(X)$ and $B_{1}(X)$ are sequentially separable, but $U S C_{p}^{f}(X)$ is not.

Proof. Let $X=Z^{\aleph_{0}}$ where $Z=\mathbb{N} \cup\{p\}$ for $p \in \mathbb{N}^{*}=\beta \mathbb{N} \backslash \mathbb{N}$.

Assume that $U S C_{p}^{f}(X)$ is sequentially separable. Then, by Theorem 3.2 , there exists a condensation $f: X \mapsto Y$ from the space $X$ on a separable metrizable space $Y$, such that $f(D)$ is an $F_{\sigma}$-set of $Y$ for any $Z_{\sigma}$-set $D$ of $X$. Since $Z$ is a continuous image of $\mathbb{N}, X$ is a continuous image of irrational numbers $\mathbb{P}$, i.e. there is a continuous mapping $\alpha: \mathbb{P} \mapsto X$ from $\mathbb{P}$ onto the space $X$. It follows that $f \circ \alpha: \mathbb{P} \mapsto Y$ is a continuous mapping, and hence $Y$ is an analytic space. By Theorem $\left[3.6, f^{-1}: Y \mapsto X\right.$ is a piecewise continuous function (i.e. $Y$ admits a closed and disjoint cover $\mathcal{F}=\left\{F_{n}: n \in \mathbb{N}\right\}$, such that for each $F_{n} \in \mathcal{F}$ the restriction $f^{-1} \mid F_{n}$ is continuous function). It follows that $f^{-1} \mid F_{n}: F_{n} \mapsto f^{-1}\left(F_{n}\right)$ is a homeomorphism, and hence $X=\bigcup_{F_{n} \in \mathcal{F}} f^{-1}\left(F_{n}\right)$ where $f^{-1}\left(F_{n}\right)$ is a separable metrizable space for each $n \in \mathbb{N}$. Since non-empty open set of $X$ is not metrizable, $f^{-1}\left(F_{n}\right)$ is a closed nowhere dense subset of $X$ for each $n \in \mathbb{N}$. But $X$ is a Baire space, a contradiction.

\section{Open questions}

Question 1. Suppose that $B_{1}(X)$ is sequentially separable. Is then $C_{p}(X)$ sequentially separable?

Question 2. Suppose that $B_{1}(X)$ is sequentially separable. Is then exist first-level Baire isomorphism $F: X \rightarrow M$ between $X$ and a separable metrizable space $M$ ? 
Question 3. Suppose that $f: X \mapsto Y$ is a first-level Baire isomorphism between $X$ and a separable metrizable space $Y$. Is then $C_{p}(X)$ sequentially separable?

Acknowledgment. The authors are grateful to Sergey V. Medvedev and the anonymous referee for making several suggestions which improved this paper.

\section{References}

[1] M.Kačena, L. Motto Ros, and B. Semmes, Some observations on "A new proof of a theorem of Jayne and Rogers", Real Analysis Exchange, 38:1 (2012/2013), 121-132.

[2] N. Noble, The density character of functions spaces, Proc. Amer. Math. Soc., 42:1 (1974), 228-233.

[3] A.V. Osipov, Application of selection principles in the study of the properties of function spaces, Acta Math. Hungar., 154:2 (2018), 362-377.

[4] A.V. Osipov, E.G. Pytkeev, On sequential separability of functional spaces, Topology Appl., 221 (2017), 270-274.

[5] A.V. Pestriakov, O prostranstvah berovskih funktsii, Issledovanij po teorii vipuklih mnogestv i grafov, Sbornik nauchnih trudov, Sverdlovsk, Ural'skii Nauchnii Center, (1987), 53-59.

[6] C.A. Rogers, J.E. Jayne, et al., Analytic Sets, Academic Press, 1980.

[7] G. Tironi, R. Isler, On some problems of local approximability in compact spaces, In : General Topology and its Relations to Modern Analysis and Algebra, III, Prague, August 30 - September 3, 1971, Academia, Prague, 1972, 443-446.

[8] N.V. Velichko, On sequential separability, Math. Notes, 78:5 (2005), 610614 .

[9] A. Wilansky, How separable is a space?, Amer. Math. Monthly, 79:7 (1972), 764-765. 